\title{
Evaluation of Laplacian Diaphragm Electromyographic Recordings in a Static Inspiratory Maneuver
}

\author{
L. Estrada ${ }^{1,2,3}$, A. Torres ${ }^{1,2,3}$, J. Garcia-Casado ${ }^{4}$, Y. Ye-Lin ${ }^{4}$ and R. Jané , $^{1,2,3}$ \\ ${ }^{1}$ Institut de Bioenginyeria de Catalunya (IBEC), Biomedical Signal Processing and Interpretation Group, Barcelona, Spain \\ ${ }^{2}$ Universitat Politècnica de Catalunya, Department d'Enginyeria de Sistemes, Automàtica i Informàtica Industrial, Barcelona, Spain \\ ${ }^{3}$ Centro de Investigación Biomédica en Red de Bioingeniería, Biomateriales y Nanomedicina (CIBER-BBN), Spain \\ ${ }^{4}$ Universitat Politècnica de València, Grupo de Bioelectrónica, Valencia, Spain
}

\begin{abstract}
Diaphragm electromyography (EMGdi) provides important information on diaphragm activity, to detect neuromuscular disorders of the most important muscle in the breathing inspiratory phase. EMGdi is habitually recorded using needles or esophageal catheters, with the implication of being invasive for patients. Surface electrodes offer an alternative for the non-invasive assessment of diaphragm activity. Ag/AgCl surface disc electrodes are used in monopolar or bipolar configuration to record EMGdi signals. On the other hand, Laplacian surface potential can be estimated by signal recording through active concentric ring electrodes. This kind of recording could reduce physiological interferences, increase the spatial selectivity and reduce orientation problems in the electrode location. The aim of this work is to compare EMGdi signals recorded simultaneously with disc electrodes in bipolar configuration and a Laplacian ring electrode over chest wall. EMGdi signal was recorded in one healthy subject during a breath hold maneuver and a static inspiratory maneuver based on Mueller's technique. In order to estimate the covered frequency range and the degree of noise contamination in both bipolar and Laplacian EMGdi signals, the cumulative percentage of the power spectrum and the signal to noise ratio in subbands were determined. Furthermore, diaphragm fatigue was evaluated by means of amplitude and frequency parameters. Our findings suggest that Laplacian EMGdi recording covers a broader frequency range although with higher noise contamination compared to bipolar EMGdi recording. Finally, in Laplacian recording fatigue indexes showed a clearer trend for muscle fatigue detection and also a reduced cardiac interference, providing an alternative to bipolar recording for diaphragm fatigue studies.
\end{abstract}

Keywords - Laplacian electrode, diaphragm muscle, fatigue, surface electromyography.

\section{INTRODUCTION}

Monitoring of muscular activity of respiratory system is an important issue for detecting medical problems, which compromise the normal ventilation process. The breathing consists on an inspiratory phase when air is inhaled and an expiratory phase when air is exhaled. During inspiration, respiratory muscles contribute to expand the thorax, move air into the lungs and develop a negative pressure. In con- trast, during expiration, muscles relax, the thorax returns to its starting position and the air is forced out from the lungs. The diaphragm, a large, dome-shaped structure, is the primary muscle involved in the inspiratory phase. Evaluation of inspiratory muscle function is essential to study respiratory diseases as chronic obstructive pulmonary disease, which increases the airway resistance to airflow and compromise the normal function of diaphragm [1]. Respiratory muscles' activity can be assessed by means of intraesophageal electrodes [2] and intramuscular electrodes [3]. However, these techniques have the disadvantage of being invasive and cumbersome for patients [4] with the potential risk of an iatrogenic pneumothorax [5]. Surface electromyography (sEMG), a non-invasive diagnostic tool for measuring muscle electrical activity, is a practical alternative for evaluation of muscle diaphragm function. sEMG is habitually collected by silver/silver chloride $(\mathrm{Ag} / \mathrm{AgCl})$ disc electrodes in monopolar or bipolar configuration. On the other hand, Laplacian of surface potential, the second derivative of the surface potential, can be evaluated by means of so-called Laplacian electrodes, a bipolar or tripolar coaxial ring electrodes. It overcomes the reduced spatial resolution and the influence of cardiac activity, drawbacks found in bipolar recordings [6, 7]. The use of Laplacian electrodes has proven promising results in the study of electrocardiogram (ECG) [6], electroencephalogram [8], electroenterogram [7], and sEMG in biceps brachii [9].

This study was undertaken to characterize the diaphragm electromyography (EMGdi) acquired simultaneously by a Laplacian electrode and $\mathrm{Ag} / \mathrm{AgCl}$ disc electrodes in bipolar configuration over diaphragm muscle. A breath hold and a static inspiratory maneuver were performed.

\section{Materials AND METHOdS}

\section{A. Subject and experimental protocol}

A nonsmoking subject with no clinical evidence of respiratory disease was included in the investigation. The subject was instructed to sit down in a comfortable chair and to stand straight with the arms beside the body, not to move 
nor talk, to breathe via a mouthpiece connected to a T-tube, and wore a disposable nose clip to prevent any air exchange through the nostrils. The conducted study consists on the EMGdi signal acquisition during a breath hold maneuver (BHM) and a static inspiratory maneuver (SIM), based on the Mueller's technique [10], to activate the diaphragm in response to a voluntary force inspiration with the T-tube occluded with only a small leak to prevent glottic closure during inspiration maneuver [4]. During the BHM, a disruption of breathing activity was performed for $15 \mathrm{~s}$. Next, during the SIM, the subject was asked to exert three maximal inspiratory pressures (MIP) during $2 \mathrm{~s}$, with 1-min resting intervals. The highest value was taken. Afterward, the subject performed a test exerting a pressure of 80 percent of the chosen MIP for $10 \mathrm{~s}$, and was encouraged to maintain the target pressure using visual feedback.

\section{B. Signal Acquisition}

Before placing the electrodes, the skin in the diaphragm area (thoracic region) was mildly abraded with gel (Nuprep, Weaver and Company, USA) and cleansed with alcohol to improve contact impedance. Pre-gelled, disposable circular electrodes of $10 \mathrm{~mm}$ diameter (foam electrode 50/PK EL501, Biopac Systems Inc, Santa Barbara, CA, USA) were placed over diaphragm muscle area between the $7^{\text {th }}$ and $8^{\text {th }}$ intercostal space, lateral to the right midclavicular line, with $50 \mathrm{~mm}$ inter-electrode distance. Dry Laplacian electrode (tripolar concentric ring electrode in quasi-bipolar configuration, TCB) of $24 \mathrm{~mm}$ outer diameter was plugged into a signal conditioner circuit and placed in the inter-electrode space of two $\mathrm{Ag} / \mathrm{AgCl}$ electrodes and attached with an adhesive plaster to skin (Fig 1), as it was presented in [9]. A reference electrode was positioned at left ankle. Bipolar electrode configuration and Laplacian electrode were plugged into an amplifier module (EMG 100C, Biopac Systems Inc, Santa Barbara, CA, USA) using a gain of 500. Moreover, Lead-I ECG was collected with an amplifier (ECG 100C, Biopac Systems Inc, Santa Barbara, CA, USA) using a gain of 500. Inspiratory mouth pressure was measured using a mouth-piece attached to a T-tube that was connected to a differential pressure transducer (TSD160,

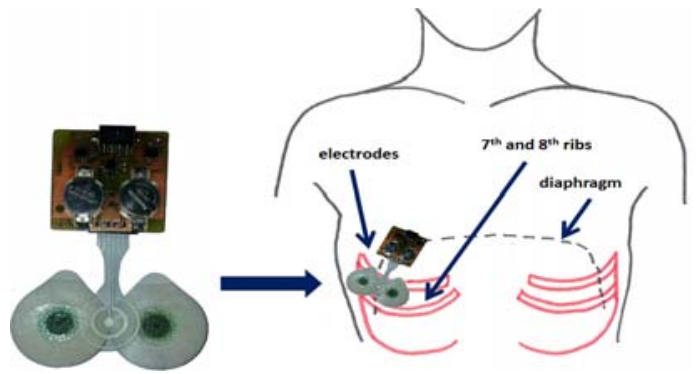

Fig. 1 (a) Two disc electrodes and Laplacian electrode (middle) for recording of EMGdi signal in diaphragm. (b) Electrode placement between

$7^{\text {th }}$ and $8^{\text {th }}$ intercostal spaces, lateral to the right midclavicular line
Biopac Systems Inc, Santa Barbara, CA, USA) plugged into a differential amplifier (DAC100C, Biopac Systems Inc, Santa Barbara, CA, USA) using a gain of 50. Amplifiers were connected to an acquisition system (MP150, Biopac Systems Inc, Santa Barbara, CA, USA) and interfaced with a computer to monitoring signals in real time and stored (AcqKnowledge software v.3.2, Santa Barbara, CA, USA). The sampling frequency used was $1000 \mathrm{~Hz}$.

\section{Signal Processing and Data Analysis}

Both bipolar and Laplacian EMGdi signals were bidirectionally bandpass filtered using a fourth-order, digital Butterworth filter with cut-off frequencies of 10 and $300 \mathrm{~Hz}$.

Estimation of the power spectral density was performed by applying the Welch modified periodogram method (Hamming window, 0.2 seconds length, 4096 - point FFT, and $50 \%$ overlap) in segments of half a second length. For the subsequent signal analysis of EMGdi power spectrum was chosen between $f_{1}=10$ to $f_{2}=300 \mathrm{~Hz}$.

Signal-to-Noise Ratio (SNR) was evaluated to compare the noise content in bipolar and Laplacian EMGdi spectrums.

SNR was calculated using the energy content in 5-second segment of SIM $\left(E_{\mathrm{I}}\right)$ and BHM $\left(E_{0}\right)$, respectively, in bands of $10 \mathrm{~Hz}$.

$$
S N R=10 \log _{10}\left(\frac{E_{I}}{E_{0}}\right)
$$

Cumulative percentage of the power distribution (CmPS), expressed as the running total of power spectrum (PS) over the total power spectrum, was used to compare the spectral distribution of bipolar and Laplacian EMGdi spectrum.

$$
\operatorname{CmPS}(f)=\frac{\int_{f_{1}}^{f} P S(f) d f}{\int_{f_{1}}^{f_{2}} P S(f) d f} \cdot 100
$$

To quantify the evolution of diaphragm muscle fatigue, we calculated the following amplitude and frequency parameters:

1) Averaged Rectified Value (ARV), an amplitude parameter, defined as average of the absolute value of the EMGdi signal, which gives an estimation of the total amount of activity over a specific time period $T$.

$$
A R V=\frac{1}{T} \int_{0}^{T}|E M G d i(t)| d t
$$

2) Median Frequency (MDF), a frequency parameter that divides the EMGdi power spectral density in two regions having the same amount of power.

$$
\int_{f_{1}}^{M D F} P S(f) \cdot d f=\int_{M D F}^{f_{2}} P S(f) \cdot d f
$$

3) Dimitrov's index, that calculates the ratio of spectral moment of order ( -1$)$ and spectral moment of order (5), to highlight lower and higher frequencies respectively, in muscle fatigue. 


$$
F \operatorname{Insm} 5=\frac{\int_{f_{1}}^{f_{2}} f^{-1} \cdot P S(f) \cdot d f}{\int_{f_{1}}^{f_{2}} f^{5} \cdot P S(f) \cdot d f}
$$

Data was analyzed using a moving window of half a second length with steps of 50ms. The signal processing was performed in MATLAB (v. R2011b, Natick, Massachusetts, USA).

\section{REsUlts}

EMG recording of diaphragm muscle is mostly affected by cardiac activity. Fig 2 shows an excerpt of a 5-sec segment of EMGdi signal, corresponding to a BHM and a SIM, picked up simultaneously by a bipolar disc-electrode configuration and a Laplacian electrode. It is evident that ECG interference greatly affects bipolar EMGdi recording (Fig 2 (a) and (b)) in comparison to Laplacian EMGdi recording (Fig 2 (c) and (d)) during both BHM (diaphragm at rest) and a SIM (high activity of diaphragm). As it is shown in Fig 3, SNR for bipolar recording is higher than Laplacian recording. However, in contrast to that of Laplacian signal, SNR of bipolar signal shows a pronounced decrease in the frequency bandwidth of the ECG components (below $30 \mathrm{~Hz}$ ). A comparative analysis of the CmPS is shown in Fig 4. Fig 4(a) reveals a rapid increase and an exponential trend in CmPS of bipolar recording during both the BHM and the SIM. The area between the breath hold and the static inspiratory maneuver for CmPS curves of bipolar recordings is greater below $50 \mathrm{~Hz}$, where the cardiac activity is present. In Fig 4(b), at rest (BHM), CmPS of Laplacian signal showed a linear trend; reflecting a spectral distribution similar to white noise. In contrast, from the same figure, it is also clear that the area between the BHM and the SIM for the curves of Laplacian signal is greater above $50 \mathrm{~Hz}$, in comparison to those of bipolar recording. In respect to diaphragm muscle fatigue, ARV, MDF and FInsm5 indexes were calculated. Figs 5 (a) presents the ECG signal corresponding to the lead I. ARV, MDF and FInsm5 fatigue
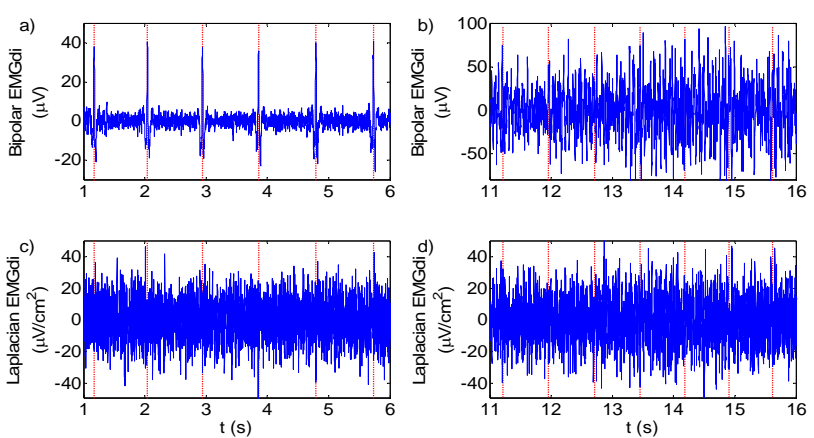

Fig. 2 An excerpt of 5-sec EMGdi recording during a breath hold maneuver and a static inspiratory maneuver: bipolar recording with disc electrodes ( $\mathrm{a}$ and $\mathrm{b}$ ) and Laplacian recording with TCB ring electrode (c and d). Vertical red dot lines indicates the $\mathrm{R}$ wave of cardiac activity

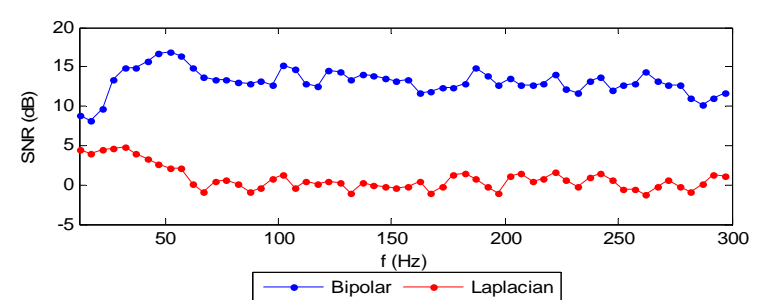

Fig. 3 Spectral distribution of SNR for bipolar and Laplacian EMGdi

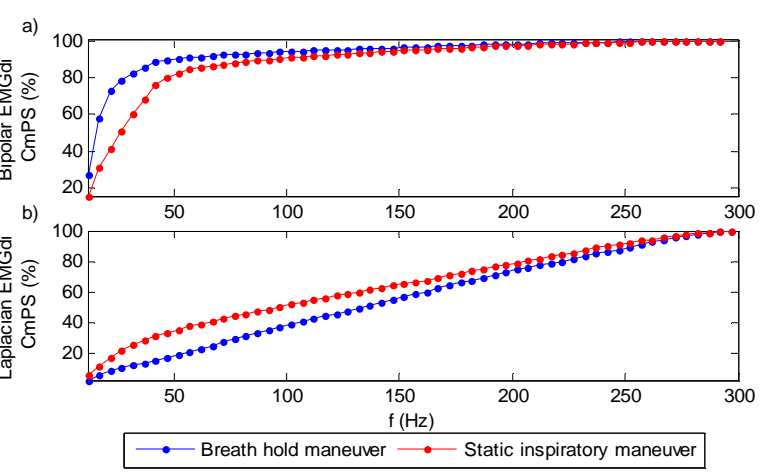

Fig. 4 Cumulative percentage of power for 5-sec EMGdi recording during a breath hold and a static inspiratory maneuver using (a) a bipolar electrode configuration and (b) a Laplacian recordings acquisition

indexes, computed from sEMG signal and recorded over the diaphragm muscle during a SIM, are shown in Fig 5 b, c and d, respectively. According to literature [11], ARV and FInsm5 increases as muscle fatigues, whereas MDF decreases. Our results show that ARV increases during SIM for both bipolar and Laplacian recording. Conversely, the FInsm5 and MDF indexes present clearer trends (rising and decreasing, respectively) in Laplacian recording in contrast to bipolar recording. In addition, the comparison of ECG in Fig 5 (a) with the evolution of fatigue indexes reveals that cardiac activity provokes a non-desired periodic oscillatory component in such evolution. Fatigue indexes in bipolar recording were more influenced by cardiac activity in comparison to Laplacian recording as shown in Fig 5 b, c and d.

\section{Discussion AND CONCLUSions}

EMGdi signal was recorded simultaneously using a bipolar disc electrode configuration and a Laplacian electrode. Previous works have reported the advantage of using Laplacian electrodes in other anatomical regions of human body [6-9]. In the present study during both BHM and SIM, power spectrum of Laplacian EMGdi signal was more distributed in frequency, contained in a broader frequency range compared to bipolar EMGdi power spectrum. Because of their physical design, Laplacian electrode presents enhanced spatial selectivity, features which are reflected in its spatial transfer function [12]. The use of a Laplacian electrode in sEMG recordings can improve the study of motor unit action potential trains $[12,13]$ and reduce the 


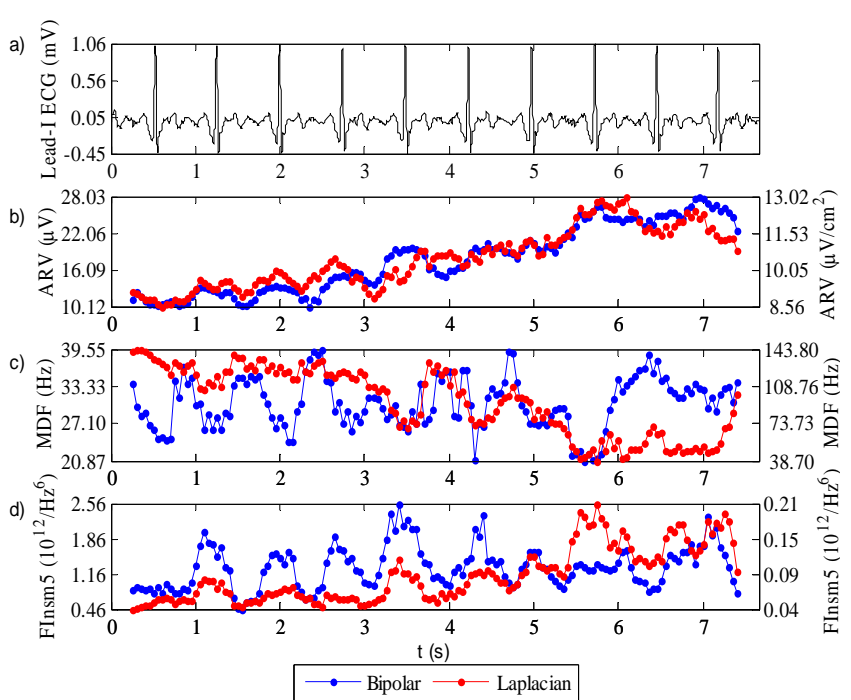

Fig. 5 (a) Lead-I ECG, (b) ARV, (c) MDF and (d) FInsm5 fatigue indexes calculated from EMGdi signal during a static inspiratory maneuver.

Left and right scales correspond to parameters from bipolar and Laplacian recordings, respectively

problem of location and orientation of the fibers related to the use of bipolar disc electrode configuration [12]. On the other hand, signal to noise ratio, was lower in Laplacian recording in comparison to bipolar recording. In this sense, it should be considered that the size of the Laplacian electrode used was smaller than the inter-electrode distance of bipolar recording with disc electrodes. The use of Laplacian electrodes of bigger diameter, or of bipolar configuration instead of TCB could yield signals of higher amplitude and better SNR.

The evolution of fatigue parameters during the SIM was similar to that reported during isometric contraction in the biceps brachii when bipolar and Laplacian electrodes were compared [9], with an increase of ARV and FInsm5 and a decrease of MDF. It was noted that ARV was influenced by cardiac activity in bipolar and Laplacian EMGdi recordings but MDF and FInsm5 were more affected in bipolar EMGdi compared to Laplacian EMGdi. Bipolar recording of EMGdi with conventional disc electrodes are severely affected by cardiac activity which provokes that most energy of this signal is concentrated below $50 \mathrm{~Hz}$.

In conclusion, this work suggests that EMGdi picked up by Laplacian electrodes is less affected by ECG interference, presenting a wider bandwidth of signal. Nevertheless, Laplacian recordings present significantly lower SNR than conventional bipolar recordings. Moreover, during the static inspiratory maneuver, trends of muscle fatigue index from Laplacian signals were much clearer and in agreement with results reported in literature. However, more subjects need to be evaluated to confirm these results and the behavior of Laplacian electrode over diaphragm in subsequent studies.

\section{AcKnOWLedgment}

The first author was supported by Instituto para la Formación y Aprovechamiento de Recursos Humanos and Secretaría Nacional de Ciencia, Tecnología e Innovación (IFARHU-SENACYT Program) from the Panama Government under grant 270-2012-273. This work was supported in part by Ministerio de Economía y Competitividad from the Spain Government under grants TEC2010-21703-C0301 and TEC2010-16945.

\section{REFERENCES}

1. Similowski T, Derenne J-PH (1994) Inspiratory muscle testing in stable COPD patients. Eur Respir J 7:1871-1876.

2. Luo YM, Moxham J, Polkey MI (2008) Diaphragm electromyography using an oesophageal catheter: current concepts. Clin Sci (Lond) 115:233-244.

3. Hodges PW, Gandevia SC (2000) Pitfalls of intramuscular electromyographic recordings from the human costal diaphragm. Clin Neurophysiol 111:1420-1424.

4. American Toracic Society/European Respitaroty Society (2002) ATS/ERS Statement on Respiratory Muscle Testing. Am J Respir Crit Care Med 166:518-624.

5. Al-Shekhlee A, Shapiro BE, Preston DC (2003) Iatrogenic complications and risks of nerve conduction studies and needle electromyography. Muscle \& Nerve 27:517-526.

6. He B, Cohen RJ (1992) Body surface Laplacian ECG mapping. IEEE Trans Biomed Eng 39:1179-1191.

7. Prats-Boluda G, Garcia-Casado J, Martinez-de-Juan JL, Ye-Lin Y (2011) Active concentric ring electrode for non-invasive detection of intestinal myoelectric signals. Med Eng Phys 33:446-55.

8. Besio WG, Koka K, Aakula R, Dai W (2006) Tri-polar concentric ring electrode development for laplacian electroencephalography. IEEE Trans Biomed Eng 53:926-933.

9. Estrada L, Torres A, Garcia-Casado J, et al. (2013) Characterization of Laplacian surface electromyographic signals during isometric contraction in biceps brachii. Proceedings of the 35th Annual International Conference of the IEEE Engineering in Medicine and Biology Society. Osaka, Japan, 2013, pp 535-538

10. Terris DJ, Hanasono MM, Liu YC (2000) Reliability of the Muller maneuver and its association with sleep-disordered breathing. Laryngoscope 110:1819-1823.

11. González-Izal M, Malanda A, Gorostiaga E, Izquierdo M (2012) Electromyographic models to assess muscle fatigue. J Electromyogr Kinesiol 22:501-512.

12. Farina D, Cescon C (2001) Concentric-ring electrode systems for noninvasive detection of single motor unit activity. IEEE Trans Biomed Eng 48:1326-1334.

13. Farina D, Arendt-Nielsen L, Merletti R, et al. (2003) Selectivity of spatial filters for surface EMG detection from the tibialis anterior muscle. IEEE Trans Biomed Eng 50:354-364.

Author: Luis Estrada

Institute: Institut de Bioenginyeria de Catalunya. Biomedical Signal Processing and Interpretation Group

Street: C/Baldiri Reixac, 4. Torre I, Planta 9

City: Barcelona

Country: Spain

Email: lestrada@ibecbarcelona.eu 